\title{
Automated higher-order calculations: Status and prospects
}

\author{
Giovanni Ossola* \\ New York City College of Technology (CUNY)
}

E-mail: gossoladcitytech.cuny.edu

In this presentation we review the current status in the automated evaluation of scattering amplitudes, with particular attention to the developments related with NLO calculations, which led to the construction of powerful multi-purpose computational tools. After a general overview, we will devote a short section to describe the GOSAM framework for NLO calculations and its application to the production of Higgs boson plus jets. We will then briefly comment on the challenges presented by NNLO calculations, whose structure is considerably more complicated. Finally, we will describe some of the features of the integrand-reduction techniques beyond NLO, an alternative promising approach to multi-loop calculations which is currently under development.

XXIII International Workshop on Deep-Inelastic Scattering

27 April - May 12015

Dallas, Texas

${ }^{*}$ Speaker. 


\section{Introduction}

The evaluation of scattering amplitudes allows us to test the theoretical models and compare their phenomenological predictions with the results of the experiments at particle colliders. In the light of the ongoing activities at the Large Hadron Collider (LHC), it is mandatory to have precise and reliable tools, that allow for an accurate and efficient evaluation of cross sections and differential distributions for a variety of processes.

In the past decade, a better understanding of the structure of scattering amplitudes was achieved thanks to the complementary work of several groups, which transformed beautiful mathematical properties of scattering amplitudes, such as recursion relations, unitarity, and integrand decomposition, into practical computational tools for the evaluation of physical observables.

In order to properly describe the data collected by the experimental collaborations, theory predictions are not reliable without accounting for higher orders, since leading-order (LO) results, usually obtained with a tree-level calculation, are affected by large theoretical errors. For most analyses, results should be provided at least at Next-to-Leading-Order precision (NLO), which are considerably more involved: they require the computation of one-loop virtual corrections (virtual part), contributions from real emission (real part), obtained by adding one additional particle in the final states, as well as a clever way of dealing with infrared divergences that occur in both virtual and real part and only cancel out when all parts are combined together. While the LO matrix elements and the NLO real parts have been available for a long time, until recently the evaluation of the virtual part of one-loop contributions represented the bottleneck towards the automation of NLO calculations. This is not the case anymore.

The scope of this talk is to summarize the recent progress in the evaluation and automation of scattering amplitudes, which led to the development of powerful automated computational tools for Next-to-Leading Order (NLO) calculations. After an overview of the many different tools which are currently available, we will devote a short paragraph to describe the GoSAM framework for NLO calculations and its application to the production of Higgs boson plus jets.

While the NLO tools are reaching their full maturity, and they are seamlessly being incorporated in the Monte Carlo programs or used to produce N-tuples of events to be used within the experimental analyses, the attention of the theoretical community is quickly shifting towards the new challenges presented by Next-to-Next-to-Leading-Order (NNLO) calculations, whose structure is considerably more complicated. We will touch on this topic during the second part of the presentation. As a development potentially relevant for future calculations beyond NLO, we will describe the extensions of the integrand reduction to higher orders in perturbation theory.

All the topics contained in this brief talk are based on a rich and extensive literature. We refer the reader to Refs. [1] (and references therein) for a more comprehensive picture of the field.

\section{Scattering Amplitudes at Next-to-Leading Order}

The standard method for the evaluation of NLO virtual corrections relies on the calculation of all the Feynman integrals associated with each process, namely to compute, for each diagram contributing to the amplitude and for each phase space point, integrals of the kind

$$
\mathscr{M}=\int d^{n} \bar{q} \mathscr{A}(\bar{q})=\int d^{n} \bar{q} \frac{\mathscr{N}(\bar{q})}{\bar{D}_{0} \bar{D}_{1} \ldots \bar{D}_{m-1}},
$$


where $\bar{D}_{i}$ are the $d$-dimensional denominators generated by the propagators of the particles inside the loop. Since any one-loop integral $\mathscr{M}$ can be decomposed in terms of a finite and known set of scalar master integrals (MIs) [2], plus an additional term $\mathscr{R}$ known in the literature as rational part, the calculation of one-loop virtual amplitudes can be summarized in terms of three separate tasks: i) the generation of the unintegrated amplitudes $\mathscr{A}$, namely their numerator functions $\mathscr{N}$ and the list of denominators $\bar{D}_{i}$; ii) the reduction of the amplitude to determine all coefficients multiplying each of the MIs and the rational term $\mathscr{R}$; iii) the evaluation of the MIs which, multiplied by the coefficients obtained in the reduction, provide the final result. Since all scalar master integrals are known and available in public codes [3] and amplitudes can be easily generated with algebraic or numerical techniques, the reduction step is what usually distinguishes the different tools available on the market.

During the past decade, the approach to one-loop calculations was revolutionized by merging the idea of four-dimensional unitarity-cuts $[4,5]$, with the understanding of the universal algebraic form of any one-loop integrals, contained in the OPP method [6]. Unitarity-based methods and integrand-level reduction techniques provided the theoretical background for development of efficient computational algorithms for NLO calculations in perturbation theory, which have been implemented in various automated codes. Tools based on generalized unitarity methods, such as Rocket [7], BLACKHAT [8], and NJET [9] have been very effective in tackling processes with high multiplicities, such as $W, Z+$ jets or multi gluon amplitudes. The traditional 4-dimensional OPP integrand reduction, implemented in the code CUTTOOLS [10] and utilized by MADLOOP [11] and HELAC-NLO [12], as well as the $d$-dimensional integrand reduction provided by SAMURAI [13] and the integrand reduction via Laurent expansion [14] implemented in NinJA [15] and used within the GoSAM framework [16] and FormCAlC [17], are instead designed to deal with several mass scales and a variety of final states. Other versatile codes are OPENLOOPS [18] and RECOLA [19], which build one-loop amplitudes numerically by means of recursion relations applied to Feynman diagrams and off-shell currents, then reduced by means of COLLIER [20]. These codes were recently employed for applications involving QCD and EW corrections.

The automated computation of physical observables at NLO accuracy, such as cross sections and differential distribution, requires to incorporate the one-loop results for the virtual amplitudes within a Monte Carlo framework (MC). In several recent applications, the MC provides the possibility of merging multiple NLO parton-level matrix elements with parton showers. For more details on the MC tools, we refer the reader to the talks of S. Prestel and F. Siegert at this Conference.

\section{Higgs boson production in Gluon Fusion with GoSam 2.0}

The GoSAM [16] framework combines automated diagram generation and algebraic manipulation [21] with the integrand reduction techniques and tensorial reduction. After the generation, the default reduction employed by GOSAM is the integrand reduction via Laurent expansion provided by NiNJA. Alternatively, the tensorial decomposition provided by GoLEM95C [22] or the $d$-dimensional integrand reduction as implemented in SAMURAI are also available.

The code has been employed in numerous applications at NLO QCD accuracy and studies of BSM scenarios (see Ref. [23] for a summary), within electroweak calculations [24], and recently 
also within NNLO calculations for the production of real-virtual contributions [25]. To achieve these results, GoSAM has been interfaced within MC tools (a detailed discussion on this topic can be found in Ref. [26]).

As an example of application, we briefly describe the efforts that led to the calculation of NLO QCD corrections to the associated production of a Higgs boson and three jets at the LHC in gluon fusion in the large top-mass limit [27]. In this limit, the Higgs coupling to gluons mediated by a top-quark loop can be described by an effective operator, leading to new Feynman rules which contains vertices involving the Higgs field and up to four gluons. Such vertices lead to Feynman integral whose rank exceeds the number of denominators. A first improvement in GoSAM needed by this calculation was the upgrade of all reduction algorithms $[14,15,28]$ to cope with additional powers of the integration momentum in the numerator functions. As a warm-up process, we tested the algorithm by computing $p p \rightarrow H+2$ jets in gluon fusion [29].

In order to deal with the complexity level of calculations such as $p p \rightarrow H+3$ jets, the GoSAM code has been further enhanced. This calculation is indeed challenging both on the side of realemission contributions and of the virtual corrections, which alone involve more than ten thousand one-loop Feynman diagrams with up to rank-seven hexagons. The introduction of numerical polarization vectors and the option to sum diagrams sharing the same propagators algebraically during the generation of the code led to an enormous gain in generation time and reduction of code size. Moreover, improvements in the performance have been achieved by exploiting the optimized algebraic manipulation of FORM 4.0. Concerning the reduction, the use of NINJA led to a faster and more stable extraction of all needed coefficients.

An updated analysis appeared in [30], which contains new results and distributions for $H+3$ jets at NLO for a set of ATLAS-like cuts and a comparison with the NLO predictions for $H+2$ jets. Very recently, new phenomenological analyses have been presented [31] which include numerical results for a large variety of observables for both standard cuts and VBF selection cuts.

\section{Beyond NLO}

The Next-to-Next-to-Leading-Order (NNLO) computations are quite far from automation and only a few computations are available for processes at hadron colliders. For a detailed discussion, we refer to the presentation of F. Petriello in the plenary session.

At one-loop, the advantage of knowing that one complete basis of MIs is formed by scalar one-loop functions and the availability of their analytic expression allowed the community to focus on the development of efficient algorithms for the extraction of the coefficients multiplying each MI. At higher-loop, a general basis of MIs is not known and they are only identified at the end of the reduction procedure. Moreover, many MIs do not have a known analytic expression and they should be evaluated numerically. The multi-loop reduction technique which is most often employed is the well-known Laporta algorithm [32], based on the solution of algebraic systems of equations obtained through integration-by-parts identities [33].

Recently, new ideas and techniques [34], along with improved version of known algorithms, are make a huge impact, paving the road to increasingly complex NNLO calculations. The progress in multi-loop calculations and in the computation of Feynman integrals using differential equations are nicely reviewed in the lectures of Refs. [35]. As of now, it is not clear to what extent we will be 
able to push the available approaches before the computational resources needed become overbearing. In this context, it will be also interesting to observe whether the extensions of integrand-level techniques to higher orders will succeed to provide a reliable alternative option.

\subsection{Integrand-Reduction Techniques Beyond One-Loop}

The reduction at the integrand level is based on the algebraic decomposition of the numerator function $\mathscr{N}$ of Eq. (2.1) in terms of the propagators in the loop, in order to identify before integration the structures that will generate the MIs, as well as terms that will vanish upon integration of the loop momentum but are needed to establish an identity for the integrands. In this approach, the coefficients in front of the MIs can be determined by solving a system of algebraic equations that are obtained by the numerical evaluation of the numerator of the integrand at explicit values of the loop-variable. The integrand reduction algorithm has been extremely successful for one-loop calculation, and it is the engine within many of the computational tools mentioned in Section 2.

Extensions beyond one-loop, first proposed in [36], have become the topic of several studies [37], thus providing a new direction in the study of multi-loop amplitudes.

Higher-loop techniques require a proper parametrization of the residues at the multi-particle poles [36]. As in the one-loop case, the parametric form of each polynomial residues is processindependent and can be determined once for all from the corresponding multiple cut. However, at higher loops, the basis of MIs is more complicated and so is the form of the residues.

In Refs. [38-40], the determination of the residues at the multiple cuts has been systematized as a problem of multivariate polynomial division in algebraic geometry. The use of these techniques proved that the integrand decomposition is applicable not only at one loop, as originally formulated, but at any order in perturbation theory. The shape of the residues is uniquely determined by the on-shell conditions, without any additional constraint. Moreover, we presented [39] a recurrence relation which, independently of the number of loops, leads to the multi-particle pole decomposition of the integrands of the scattering amplitudes. Applications to two-loop Feynman diagrams in QED and QCD showed that the proposed reduction algorithm can be applied to integrands with denominators appearing with arbitrary powers [40].

\section{Summary and Conclusions}

Scattering amplitudes provide an ideal testing ground for many theoretical applications. A better understanding of the mathematical properties of scattering amplitudes indeed allows for the construction of efficient algorithms for their evaluation, and ultimately leads to higher quality theoretical predictions to be used in the experimental analyses at particle colliders.

There is a variety of approaches and numerical tools available for one-loop calculations, which are interfaced with Monte Carlo event generators to provide NLO predictions for processes needed by the LHC experimental collaborations. Just like their tree-level predecessors, these codes allow the user to compute full NLO calculations at the simple effort of providing the list of particles and some input parameters.

Looking ahead, the focus is shifting towards the challenges presented by NNLO calculations, which are considerably more involved. While a full automation of NNLO is still not around the corner, there are plenty of activities and studies in the making, and the progress in the field is 
tangible, both in terms of the development of new ideas and techniques and the completion of new calculations and phenomenological studies.

Acknowledgments Work supported in part by the National Science Foundation under Grants PHY-1068550 and PHY-1417354. We also acknowledge PSC-CUNY Award No. 67536-00 45.

\section{References}

[1] Bern Z, Dixon L J and Kosower D A 2007 Annals Phys. 322 1587-1634 (Preprint 0704.2798); Ellis R K, Kunszt Z, Melnikov K and Zanderighi G 2012 Phys.Rept. 518 141-250 (Preprint 1105.4319); Ossola G 2014 J.Phys.Conf.Ser. 523012040 (Preprint 1310.3214); Mastrolia P 2015 (Preprint 1507.03226)

[2] Passarino G and Veltman M J G 1979 Nucl. Phys. B160 151; 't Hooft G and Veltman M 1979 Nucl.Phys. B153 365-401

[3] van Oldenborgh G 1991 Comput.Phys.Commun. 66 1-15; Hahn T and Perez-Victoria M 1999 Comput.Phys.Commun. 118 153-165 (Preprint hep-ph/9807565); Ellis R K and Zanderighi G 2008 JHEP 02002 (Preprint 0712.1851); van Hameren A 2011 Comput.Phys.Commun. 182 2427-2438 (Preprint 1007.4716); Cullen G, Guillet J, Heinrich G, Kleinschmidt T, Pilon E et al. 2011 Comput.Phys.Commun. 182 2276-2284 (Preprint 1101.5595)

[4] Bern Z, Dixon L J, Dunbar D C and Kosower D A 1994 Nucl. Phys. B425 217-260 (Preprint hep-ph/9403226)

[5] Britto R, Cachazo F and Feng B 2005 Nucl. Phys. B725 275-305 (Preprint hep-th/0412103)

[6] Ossola G, Papadopoulos C G and Pittau R 2007 Nucl.Phys. B763 147-169 (Preprint hep-ph/0609007); Ossola G, Papadopoulos C G and Pittau R 2007 JHEP 0707085 (Preprint 0704.1271); Ossola G, Papadopoulos C G and Pittau R 2008 JHEP 0805004 (Preprint 0802.1876); Mastrolia P, Ossola G, Papadopoulos C G and Pittau R 2008 JHEP 06030 (Preprint 0803.3964)

[7] Giele W and Zanderighi G 2008 JHEP 0806038 (Preprint 0805.2152); Ellis R, Giele W T, Kunszt Z and Melnikov K 2009 Nucl.Phys. B822 270-282 (Preprint 0806.3467)

[8] Berger C, Bern Z, Dixon L, Febres Cordero F et al. 2008 Phys.Rev. D78 036003 (Preprint 0803.4180)

[9] Badger S, Biedermann B, Uwer P and Yundin V 2013 Comput.Phys.Commun. 184 1981-1998 (Preprint 1209.0100)

[10] Ossola G, Papadopoulos C G and Pittau R 2008 JHEP 03042 (Preprint 0711.3596)

[11] Hirschi V, Frederix R, Frixione S, Garzelli M V et al. 2011 JHEP 1105044 (Preprint 1103.0621)

[12] Bevilacqua G, Czakon M, Garzelli M, van Hameren A, Kardos A et al. 2013 Comput.Phys.Commun. 184 986-997 (Preprint 1110.1499)

[13] Mastrolia P, Ossola G, Reiter T and Tramontano F 2010 JHEP 1008080 (Preprint 1006.0710)

[14] Mastrolia P, Mirabella E and Peraro T 2012 JHEP 1206095 (Preprint 1203.0291)

[15] van Deurzen H, Luisoni G, Mastrolia P, Mirabella E, Ossola G et al. 2014 JHEP 1403115 (Preprint 1312.6678); Peraro T 2014 Comput.Phys.Commun. 185 2771-2797 (Preprint 1403.1229)

[16] Cullen G, Greiner N, Heinrich G, Luisoni G, Mastrolia P et al. 2012 Eur.Phys.J. C72 1889 (Preprint 1111.2034); Cullen G, van Deurzen H, Greiner N, Heinrich G, Luisoni G et al. 2014 Eur.Phys.J. C74 3001 (Preprint 1404.7096) 
[17] Nejad B C, Hahn T, Lang J N and Mirabella E 2013 (Preprint 1310.0274)

[18] Cascioli F, Maierhofer P and Pozzorini S 2012 Phys.Rev.Lett. 108111601 (Preprint 1111.5206)

[19] Actis S, Denner A, Hofer L, Scharf A and Uccirati S 2013 JHEP 1304037 (Preprint 1211.6316)

[20] Denner A, Dittmaier S and Hofer L 2014 PoS LL2014 071 (Preprint 1407.0087)

[21] Nogueira P 1993 J.Comput.Phys. 105 279-289; Vermaseren J A M 2000 (Preprint math-ph/0010025); Reiter T 2010 Comput.Phys.Commun. 181 1301-1331 (Preprint 0907.3714); Cullen G, Koch-Janusz M and Reiter T 2011 Comput.Phys.Commun. 182 2368-2387 (Preprint 1008.0803); Kuipers J, Ueda T, Vermaseren J and Vollinga J 2013 Comput.Phys.Commun. 184 1453-1467 (Preprint 1203.6543)

[22] Binoth T, Guillet J P, Heinrich G, Pilon E and Reiter T 2009 Comput.Phys.Commun. 180 2317-2330 (Preprint 0810.0992); Heinrich G, Ossola G, Reiter T and Tramontano F 2010 JHEP 1010105 (Preprint 1008.2441)

[23] Greiner N 2014 (Preprint 1410.3237)

[24] Chiesa M, Greiner N and Tramontano F 2015 (Preprint 1507.08579)

[25] Gao J and Zhu H X 2014 Phys.Rev. D90 114022 (Preprint 1408.5150); Gao J and Zhu H X 2014 Phys.Rev.Lett. 113262001 (Preprint 1410.3165); Del Duca V, Duhr C, Somogyi G, Tramontano F and Trocsanyi Z 2015 (Preprint 1501.07226)

[26] van Deurzen H, Greiner N, Heinrich G, Luisoni G et al. 2014 PoS LL2014 021 (Preprint 1407.0922)

[27] Cullen G, van Deurzen H, Greiner N, Luisoni G, Mastrolia P et al. 2013 Phys.Rev.Lett. 111131801 (Preprint 1307.4737)

[28] van Deurzen H 2013 Acta Phys.Polon. B44 2223-2230; Guillet J P, Heinrich G and von Soden-Fraunhofen J F 2014 Comput. Phys. Commun. 185 1828-1834 (Preprint 1312.3887)

[29] van Deurzen H, Greiner N, Luisoni G, Mastrolia P, Mirabella E et al. 2013 Phys.Lett. B721 74-81 (Preprint 1301.0493)

[30] Butterworth J, Dissertori G, Dittmaier S, de Florian D, Glover N et al. 2014 (Preprint 1405.1067)

[31] Greiner N, Hoeche S, Luisoni G, Schonherr M, Winter J C and Yundin V 2015 (Preprint 1506.01016)

[32] Laporta S 2000 Int.J.Mod.Phys. A15 5087-5159 (Preprint hep-ph/0102033)

[33] Tkachov F 1981 Phys.Lett. B100 65-68

[34] Henn J M 2013 Phys.Rev.Lett. 110251601 (Preprint 1304.1806); Argeri M, Di Vita S, Mastrolia P, Mirabella E, Schlenk J et al. 2014 JHEP 1403082 (Preprint 1401.2979); Papadopoulos C G 2014 JHEP 1407088 (Preprint 1401.6057); Gehrmann T, von Manteuffel A, Tancredi L and Weihs E 2014 JHEP 1406032 (Preprint 1404.4853); Lee R N 2014 (Preprint 1411.0911)

[35] Duhr C 2014 (Preprint 1411.7538); Henn J M 2015 J. Phys. A48 153001 (Preprint 1412.2296)

[36] Mastrolia P and Ossola G 2011 JHEP 1111014 (Preprint 1107.6041)

[37] Badger S, Frellesvig H and Zhang Y 2012 JHEP 1204055 (Preprint 1202.2019); Kleiss R H, Malamos I, Papadopoulos C G and Verheyen R 2012 JHEP 1212038 (Preprint 1206.4180); Badger S, Frellesvig H and Zhang Y 2012 JHEP 1208065 (Preprint 1207.2976); Feng B and Huang R 2013 JHEP 1302117 (Preprint 1209.3747); Mastrolia P, Mirabella E, Ossola G and Peraro T 2013 Phys.Rev. D87 085026 (Preprint 1209.4319)

[38] Zhang Y 2012 JHEP 1209042 (Preprint 1205.5707)

[39] Mastrolia P, Mirabella E, Ossola G and Peraro T 2012 Phys.Lett. B718 173-177 (Preprint 1205.7087)

[40] Mastrolia P, Mirabella E, Ossola G and Peraro T 2013 Phys.Lett. B727 532-535 (Preprint 1307.5832) 